INPLASY

PROTOCOL

To cite: Furquim et al. Dental regeneration through bioengineering: a systematic scoping review. Inplasy protocol 202120042. doi: 10.37766/inplasy2021.2.0042

Received: 11 February 2021

Published: 11 February 2021

Corresponding author: Camila Pinheiro Furquim

camilapfurquim@yahoo.com.br

Author Affiliation:

Guarulhos university

Support: No funding was provided.

Review Stage at time of this submission: Data analysis.

Conflicts of interest:

None declared.

\section{Dental regeneration through bioengineering: a systematic scoping review}

Furquim, CP1; Kumagai, RY2; Bustillos-Torrez, W3; Tanaka, C4; Meza-Mauricio, J5; Retamal-Valdes, B6; Shibli, JA7.

Review question / Objective: What is the state-of-the-art for the whole tooth regeneration "in vivo"?

Condition being studied: Tooth regeneration.

Information sources: The grey literature in the System for Information on Grey Literature in Europe (http:// www.opengrey.eu) and The New York Academy of Medicine Grey Literature Report (http://www.greylit.org) were screened electronically, as recommended by the high standards for systematic reviews (AMSTAR guideline). Finally, the references of included studies were explored to capture any potential additional records, as suggested by Greenhalgh and Peacock.

INPLASY registration number: This protocol was registered with the International Platform of Registered Systematic Review and Meta-Analysis Protocols (INPLASY) on 11 February 2021 and was last updated on 11 February 2021 (registration number INPLASY202120042).

\section{INTRODUCTION}

Review question / Objective: What is the state-of-the-art for the whole tooth regeneration "in vivo"?

Condition being studied: tooth regeneration.

\section{METHODS}

Search strategy: MEDLINE (pubmed), Scopus, Embase, Web of science, Bireme, Livivo and Google Scholar databases were searched up to January 2021 by two independent reviewers (C.P.F and W.B) using keywords. 
Participant or population: Does not apply.

Intervention: Does not apply.

\section{Comparator: Does not apply.}

Study designs to be included: In vivo studies.

Eligibility criteria: publications reporting the whole tooth reproduction "in vivo" by a bioengineered process with eruption in the oral cavity.

Information sources: The grey literature in the System for Information on Grey Literature in Europe (http:// www.opengrey.eu) and The New York Academy of Medicine Grey Literature Report (http://www.greylit.org) were screened electronically, as recommended by the high standards for systematic reviews (AMSTAR guideline). Finally, the references of included studies were explored to capture any potential additional records, as suggested by Greenhalgh and Peacock.

Main outcome(s): Whole tooth regeneration eruption.

Quality assessment / Risk of bias analysis: Risk of bias was not performed.

Strategy of data synthesis: Data on the following issues were extracted and recorded: citation, publication status and year of publication; the study consists in the engineering of reconstruct a whole tooth.

Subgroup analysis: Subgroup analysis was not performed.

Sensitivity analysis: Sensitivity analysis was not performed.

Country(ies) involved: Brazil.

Keywords: dental regeneration, whole tooth replacement, bioengeneer regeneration, tissue engenieering, review.
Contributions of each author:

Author 1 - Camila Pinheiro Furquim Performed the search in the database and drafted the manuscript.

Email: camilapfurquim@yahoo.com.br

Author 2 - Rose Yakushijin Kumagai Drafted the manuscript.

Author 3 - Willy Bustillos-Torrez Performed the search in the database, data collection and drafted the manuscript.

Author 4 - Caio Tanaka - drafted the manuscript.

Author 5 - Jonathan Meza-Mauricio - Data collection and drafted the manuscript.

Email: jomemau60@gmail.com

Author 6 - Belen Retamal-Valdes - Writing review and editing of the current manuscript.

Author 7 - Jamil A. Shibli - Writing review and editing of the current manuscript. 